\title{
Electroweak tests of the Standard Model
}

\author{
Jens Erler \\ Departamento de Física Teórica, Instituto de Física, Universidad Nacional Autónoma de México, \\ México D.F. 04510, México
}

\begin{abstract}
Electroweak precision tests of the Standard Model of the fundamental interactions are reviewed ranging from the lowest to the highest energy experiments. Results from global fits are presented with particular emphasis on the extraction of fundamental parameters such as the Fermi constant, the strong coupling constant, the electroweak mixing angle, and the mass of the Higgs boson. Constraints on physics beyond the Standard Model are also discussed.
\end{abstract}

Keywords: Electroweak precision data, global fit, Higgs boson mass.

PACS: 12.15.-y,14.60.-z,14.70.-e,14.80.Bn.

\section{INTRODUCTION}

The Standard Model (SM) of the electroweak (EW) interactions has been developed mostly in the 1960s, where the gauge group $S U(2)_{L} \times U(1)_{Y}$ was suggested [1], the Higgs mechanism for spontaneously broken gauge theories developed, and the model for leptons constructed explicitly [2]. Subsequently, key predictions of the SM were observed in the 1970s, including neutral currents and parity non-conservation in atoms and in deep-inelastic electron scattering [3]. The basic structure of the SM was established in the 1980s after mutually consistent values of the weak mixing angle, $\sin ^{2} \theta_{W}$, were determined from many different processes. The 1990s saw the highly successful Zfactories, LEP and SLC, and the confirmation of the SM at the loop level. It thus became clear that any new physics beyond the SM could at most be a perturbation. The previous decade added precision measurements in the neutrino and quark sectors (including a $0.5 \%$ measurement of the top quark mass [4]), as well as ultra-high precision determinations of the $W$-boson mass, $M_{W}$ (to $2 \times 10^{-4}$ ) [5], the anomalous magnetic moment of the muon [6], and the Fermi constant, $G_{F}$ [7]. These results suggest that the new physics must be separated by at least a little hierarchy from the EW scale unless one considers the possibility that a conspiracy is at work. The current decade will elucidate the EW symmetry breaking sector at the LHC and witness a new generation of experiments at the intensity frontier with sensitivities to the multi-TeV scale and beyond.

\section{RECENT DEVELOPMENTS}

The MuLan Collaboration at the PSI in Switzerland [7] has measured the $\mu$-lifetime to parts-per-million precision, $\tau_{\mu}=2.1969803(2.2) \times 10^{-6} \mathrm{~s}$, which translates into a determination of

$$
G_{F}=1.1663787(6) \times 10^{-5} \mathrm{GeV}^{-2} .
$$


The Higgs vacuum expectation value is given by $\langle 0|H| 0\rangle=\left(\sqrt{2} G_{F}\right)^{-1 / 2}=246.22 \mathrm{GeV}$. This result is so precise that even the error in the definition of the atomic mass unit $(\mathrm{u})$ can shift $G_{F}$ (MuLan quotes $G_{F}=1.1663788(7) \times 10^{-5} \mathrm{GeV}^{-2}$ ). Moreover, the effect of the finite $M_{W}$ in the W-propagator is no longer negligible. One may either correct for it, i.e., absorb it in $\Delta q$ defined through $\tau_{\mu}^{-1} \propto G_{F}^{2} m_{\mu}^{5}(1+\Delta q)$, or else [8] absorb it in $\Delta r$ [9] defined in terms of the accurately known fine structure constant, $\alpha$, and $Z$-boson mass, $M_{Z}$,

$$
\sqrt{2} G_{F} M_{W}^{2}\left(1-\frac{M_{W}^{2}}{M_{Z}^{2}}\right) \equiv \frac{\pi \alpha}{1-\Delta r} .
$$

The latter convention is motivated by an effective Fermi theory point of view, and used by MuLan and since this year also by the PDG [10].

What $\tau_{\mu}$ is to $G_{F}$ is the $\tau$-lifetime to the strong coupling constant, $\alpha_{s}$. At least one lowenergy $\alpha_{s}$-value is needed to promote the $Z$-width and related $Z$-pole observables from a quantitative measurement in QCD to an EW SM test (or to constrain physics beyond the $\mathrm{SM}$ ). Perturbative QCD has recently been extended to 4-loop order [12], but there is a controversy whether the perturbative series should be truncated (FOPT) [13] or whether higher order terms from the running strong coupling in the complex plane should be re-summed (CIPT) [14]. There are also non-perturbative contributions parametrized by condensate terms which can be constrained by experimentally determined spectral functions. There are two different approaches $[15,16]$ which at present give very similar numerical results. Using FOPT and the condensates from Ref. [16] we find,

$$
\alpha_{s}[\tau]=0.1193 \pm 0.0021, \quad \alpha_{s}[Z]=0.1197 \pm 0.0028
$$

where the latter determination from the $Z$-pole is the only extraction of $\alpha_{s}$ with a very small theory uncertainty. The two values can be seen to agree perfectly.

The most precise derived and purely EW precision observable is no longer the $Z$-pole combination of $\sin ^{2} \theta_{W}$, but rather $M_{W}=80.387 \pm 0.016 \mathrm{GeV}$ from the CDF and D $\varnothing$ Collaborations at the Tevatron [5] which is dominated by a $\pm 19 \mathrm{MeV}$ determination by CDF using only $2.2 \mathrm{fb}^{-1}$ of their data. Together with the LEP 2 combination [17], $M_{W}=80.376 \pm 0.033 \mathrm{GeV}$, one obtains for the on-shell definition of $\sin ^{2} \theta_{W}$,

$$
\sin ^{2} \theta_{W}^{\text {on-shell }} \equiv 1-\frac{M_{W}^{2}}{M_{Z}^{2}}=0.22290 \pm 0.00028,
$$

from which and can extract $M_{H}=96_{-25}^{+29} \mathrm{GeV}$. As for the updated global EW fit, we find,

$$
M_{H}=102_{-20}^{+24} \mathrm{GeV} \text {. }
$$

The prospects for the full $10 \mathrm{fb}^{-1}$ dataset are $\mathrm{a} \pm 13 \mathrm{MeV} M_{W}$ determination from CDF alone, even when no reduction of the parton distribution function $( \pm 10 \mathrm{MeV})$ and QED $( \pm 4 \mathrm{MeV})$ uncertainties is assumed. In the most optimistic scenario, CDF could shrink the error to $\pm 10 \mathrm{MeV}$, which is to be compared with the $\pm 6 \mathrm{MeV}$ accuracy expected from a threshold scan at a future International Linear Collider.

The anomalous magnetic moment of the muon was measured to extreme precision,

$$
a_{\mu} \equiv \frac{g_{\mu}-2}{2}=(1165920.80 \pm 0.63) \times 10^{-9},
$$




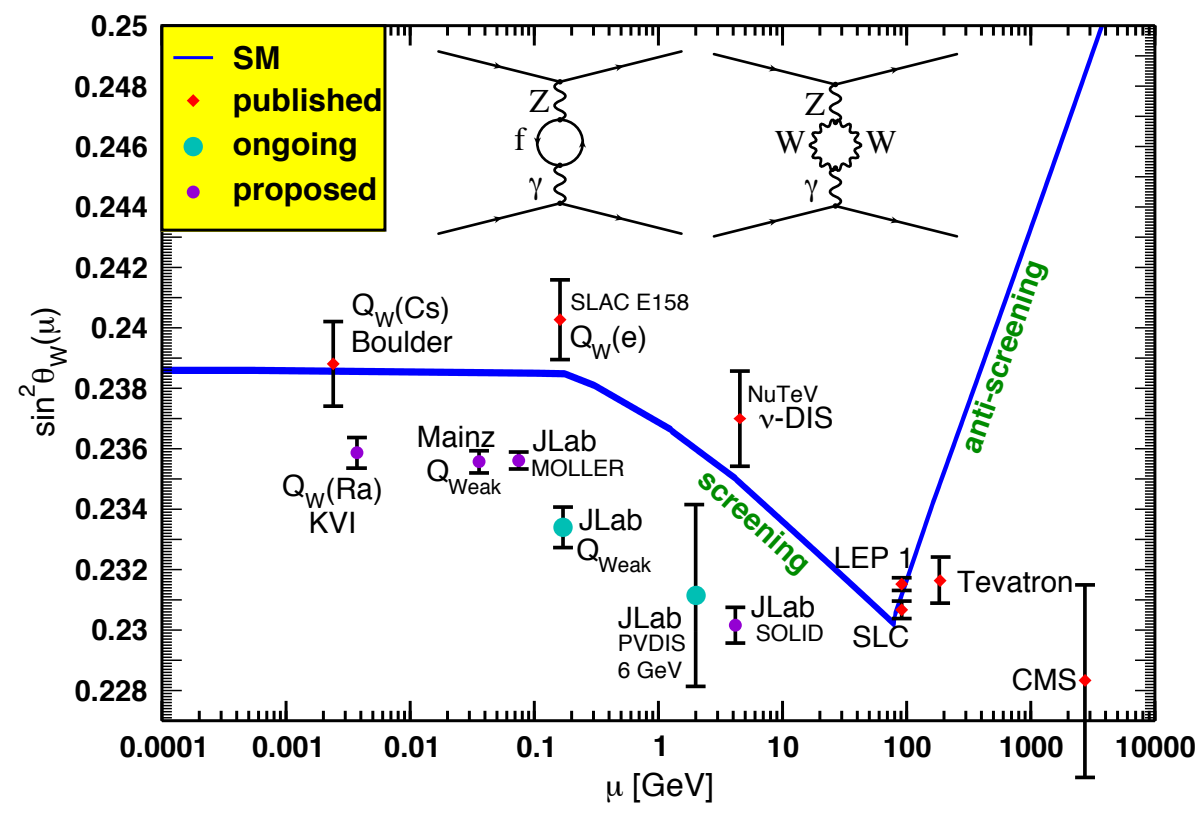

FIGURE 1. Current and future measurements of the running weak mixing angle. The uncertainty in the prediction is small except possibly in the hadronic transition region roughly between 0.1 and $2 \mathrm{GeV}$ [26]. The relevant $Q^{2}$ of the Tevatron and CMS values make them effectively additional $Z$-pole measurements, but for clarity they have been shifted horizontally to the right.

by the BNL-E821 Collaboration [6]. The prediction, $a_{\mu}=(1165918.41 \pm 0.48) \times 10^{-9}$, from the SM includes $e^{+} e^{-}$as well as $\tau$-decay data in the dispersion integral needed to constrain the two- and three-loop vacuum polarization contributions and differs by $3.0 \sigma$. The data based on $\tau$-decays requires an isospin rotation and a corresponding correction to account for isospin violating effects and suggest a smaller $(2.4 \sigma)$ discrepancy, while the $e^{+} e^{-}$-based data sets (from annihilation and radiative returns) by themselves would imply a $3.6 \sigma$ conflict. Indeed, there is a $2.3 \sigma$ discrepancy between the experimental branching ratio, $B\left(\tau^{-} \rightarrow v \pi^{0} \pi^{-}\right)$, and its SM prediction using the $e^{+} e^{-}$data [18]. In view of this, it is tempting to ignore the $\tau$-decay data and blame the difference to the $e^{+} e^{-}$data on unaccounted for isospin violating effects. However, there is also a $1.9 \sigma$ experimental conflict between KLOE and BaBar (both using the radiative return method [19]) the latter not being inconsistent with the $\tau$-data. As for the question whether the deviation in $a_{\mu}$ may arise from physics beyond the SM (especially supersymmetry), my personal take is that I am less concerned about these hadronic issues than the absence of convincing new physics hints at the Tevatron or the LHC. In any case there is an important new proposal at Fermilab to improve on the precision in Eq. (6) by a factor of four [6]. 


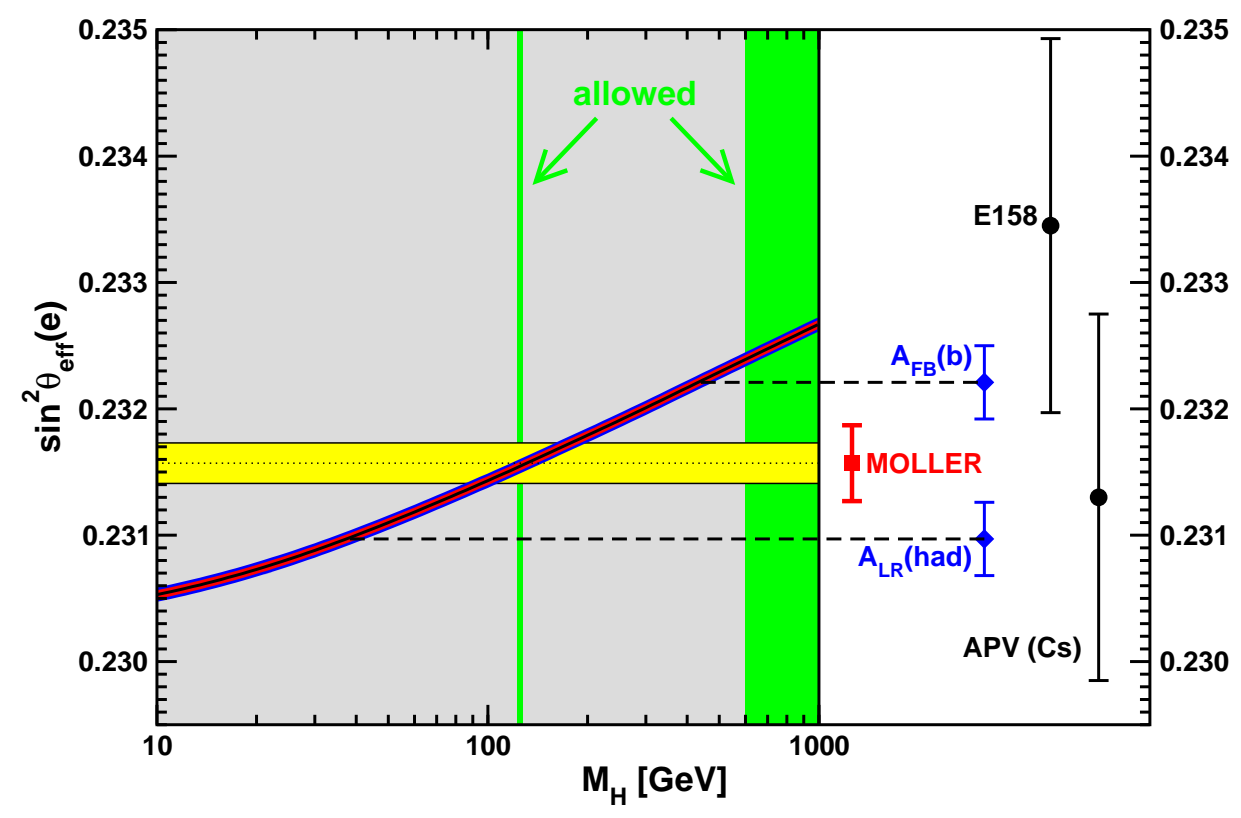

FIGURE 2. Implication of $\sin ^{2} \theta_{W}$ measurements for $M_{H}$. Shown are the most precise determinations from LEP 1 and the SLC, and the extractions from APV and from current (E158) and future (MOLLER) polarized Møller scattering. Also indicated are the non-excluded intervals from direct Higgs searches.

\section{Parity-violating electron scattering}

High precision measurements in the EW sector are also possible at the intensity frontier, when QED and QCD effects are filtered out by using parity-violating observables.

The JLab Qweak detector [20] at the $6 \mathrm{GeV}$ CEBAF was dedicated to a measurement of the weak charge of the proton, $Q_{W}^{p} \propto 1-4 \sin ^{2} \theta_{W}$, to $4 \%$ precision in elastic polarized $e^{-} p$ scattering at $Q^{2}=0.026 \mathrm{GeV}^{2}$. Data taking is complete and the analysis is in progress. $Q_{W}^{p}$ is similar to the weak charges of heavy nuclei measured in atomic parity violation (APV) but at a different kinematics. This circumstance results in a reenhancement of the $\gamma-Z$ box contribution [21] introducing an extra theory uncertainty.

MOLLER [22] is an ultra-high precision measurement of $\sin ^{2} \theta_{W}$ in polarized Møller scattering at the $12 \mathrm{GeV}$ upgraded CEBAF [23]. It aims at a factor of 5 improvement over a similar experiment at SLAC by the E158 Collaboration [24], and would be one of the worlds most precise determinations of $\sin ^{2} \theta_{W}$ and the most accurate at low energies.

PVDIS was a deep-inelastic polarized $e^{-}$scattering experiment using the $6 \mathrm{GeV}$ CEBAF and is currently in the analysis phase [25]. Together with SOLID (at $12 \mathrm{GeV}$ ) an array of kinematics points will be measured to test strong, EW, and new physics.

Fig. 1 summarizes these and other current and future (projected) determinations of $\sin ^{2} \theta_{W}$ as a function of energy scale $\mu$. 


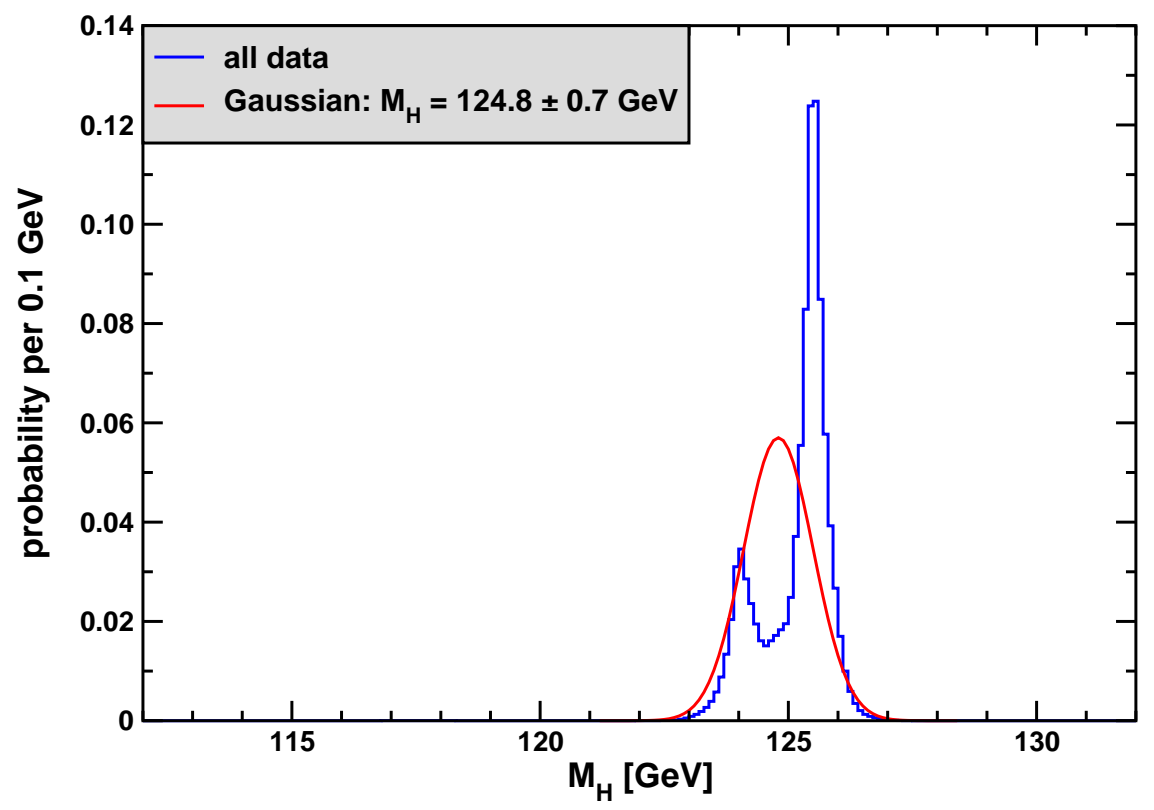

FIGURE 3. The histogram shows the normalized probability distribution of $M_{H}$. The bell shaped curve is a reference Gaussian density defined to contain the same probability as the histogram over the region of bins which are higher than the tail bins. The significance of this region corresponds to $3.4 \sigma$.

\section{SM INTERPRETATION: $M_{H}$}

The various $\sin ^{2} \theta_{W}$ measurements discussed above can be used to constrain $M_{H}$ and compare it with the results obtained at the LHC. It is important to recall that the most precise determinations at LEP 1 (from the forward-backward cross-section asymmetry of $Z$-bosons decaying into $b \bar{b}$ pairs, $A_{F B}(b)$ ) and at the SLC (from the polarization asymmetry for hadronic final states, $A_{L R}($ had)), both of which being mostly sensitive to the initial state (electron) coupling, are discrepant by three standard deviations. Their average, on the other hand, corresponds to values of $M_{H}$ that are in perfect agreement with the Higgs boson candidates seen by the ATLAS [27] and CMS [28] Collaborations at the LHC. This is illustrated in Fig. 2 together with the low-energy determinations from E158 [24] and APV which is dominated by the experiment in Cs [29] and makes use of the atomic theory calculation ${ }^{1}$ of Ref. [31].

Estimating the significance of the LHC data [27, 28] by themselves requires a "look elsewhere effect correction" which is, however, poorly defined. It can be avoided when

${ }^{1}$ After the conference had adjourned there appeared an update of the atomic structure calculation [30] finding significant corrections to formally subleading terms. Taking this into account moves the extracted Cs weak charge $1.5 \sigma$ below the SM prediction, which then favors lower values of $M_{H}$. 


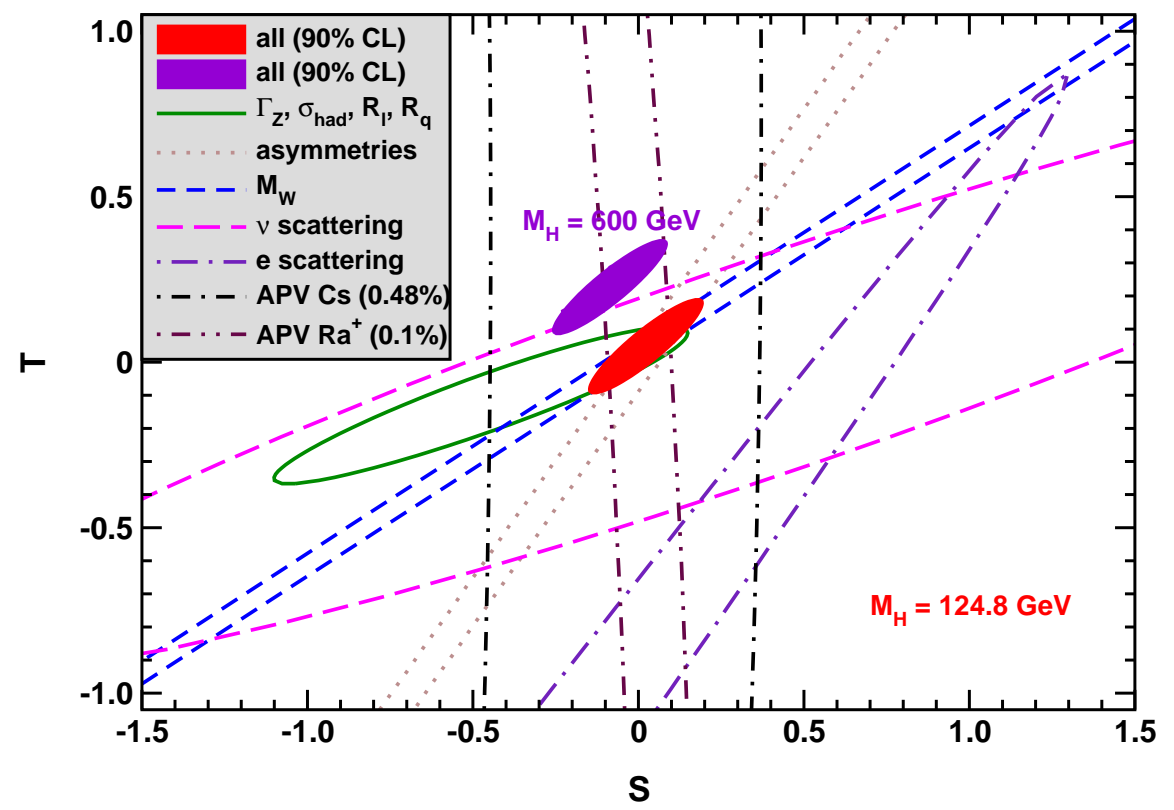

FIGURE 4. $1 \sigma$ constraints on $S$ and $T$ from various inputs. The contours assume $M_{H}=124.8 \mathrm{GeV}$ except for the upper (violet) one for all data which is for $M_{H}=600 \mathrm{GeV}$. The contour labeled APV $\mathrm{Ra}^{+}$refers to a future experiment on a single trapped $\mathrm{Ra}$ ion which is in preparation at the KVI in Groningen [40]. The atomic structure of $\mathrm{Ra}^{+}$is alkali-like so that the atomic theory parallels that of Cs, but due to its greater neutron excess Ra constrains a linear combination of $S$ and $T$ which is different from $C s$ and quite orthogonal to the $M_{W}$ and $\sin ^{2} \theta_{W}$ contours.

they are combined with the Higgs search results from LEP 2 [32] and the Tevatron [33] as well as with the EW precision data [34], the latter providing a normalizable probability distribution (shown in Fig. 3). This requires the validity of the SM which used to be a very strong assumption in the past. But with the absence of clear new physics signals at the energy frontier this can now be seen as a reasonable approximation.

\section{NEW PHYSICS INTERPRETATIONS}

The EW precision tests also set strong constrains on models of new physics. E.g., if the Higgs hints are real, an extra fermion generation is ruled out at the 99.6\% CL. [35]. This leaves us with basically three scenarios, all of which in need of some tuning and faith (the mass spectra are generally quite similar): (i) One ignores the collider bumps (or assigns them to something else) and assumes $M_{H} \lesssim 120 \mathrm{GeV}$ (see e.g., Ref. [36]); (ii) one assumes instead $M_{H} \gtrsim 450 \mathrm{GeV}$ [37]; (iii) or one accepts $M_{H} \approx 125 \mathrm{GeV}$ and introduces new physics beyond a fourth generation, such as an extra Higgs doublet [38].

More generally, whenever the new physics is rather heavy and mostly affects the 


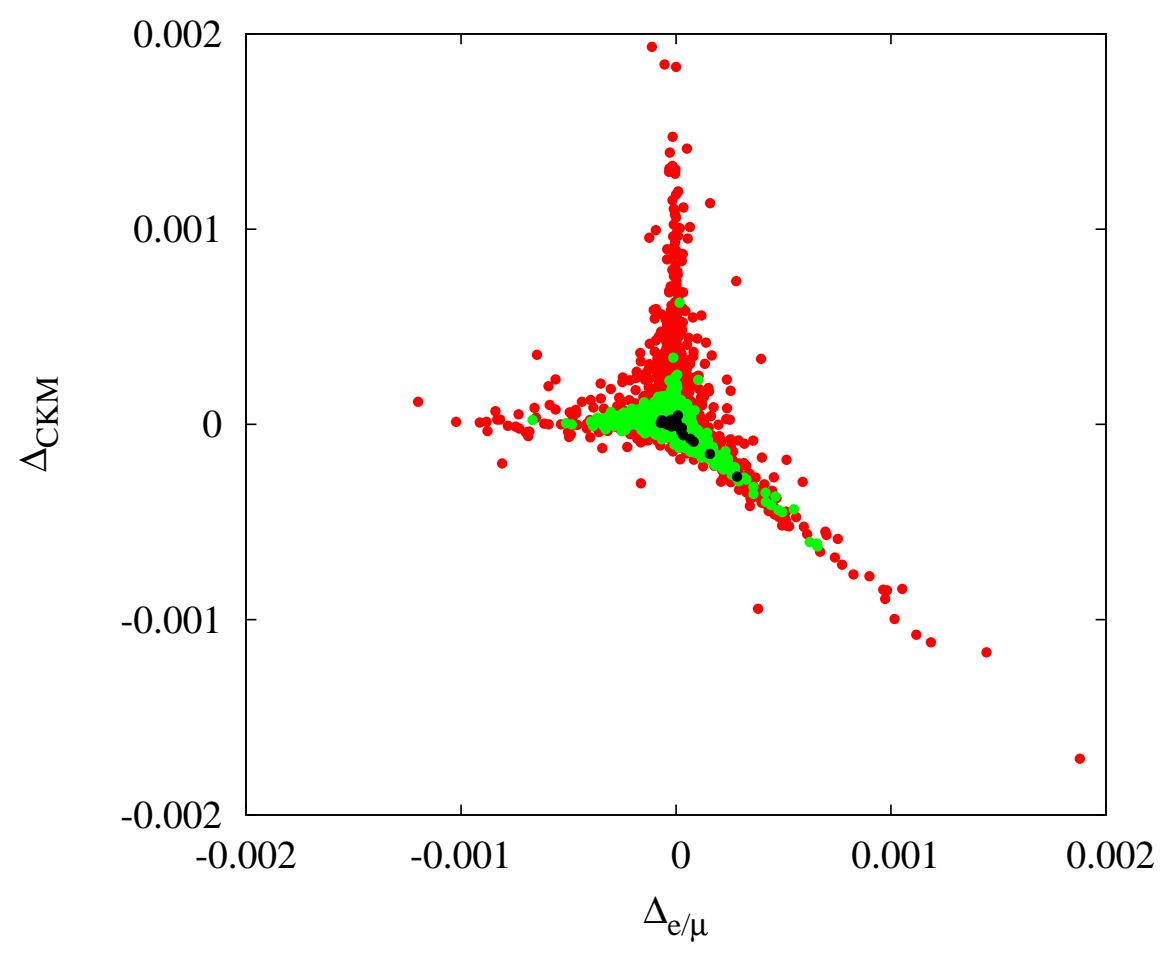

FIGURE 5. Scatter plot [41] of MSSM points satisfying the $\Delta_{\mathrm{CKM}}$ and $\Delta_{e} / \mu$ constraints. Points satisfying in addition the EW precision data including (excluding) LHC bounds are shown in black (green). $\Delta_{\mathrm{CKM}}$ is enhanced when there is a large difference between the masses of the first generation squarks and the second generation sleptons. Similarly, $\Delta_{e} \mu$ is enhanced when the first and second generation slepton masses are significantly split.

gauge boson self-energies, one can parametrize it in terms of the oblique parameters $S$ and $T$ [39] (a third parameter, $U$, is usually small). The constraints on $S$ and $T$ from various data sets are shown in Fig. 4 (where $U=0$ is assumed).

The observables discussed so far are mostly related to the weak neutral current, but tests of charged current universality can also provide information on new physics. Denoting any deviation from the unitarity of the first row of the CKM quark mixing matrix by $\Delta_{\mathrm{CKM}} \equiv\left|V_{u d}\right|^{2}+\left|V_{u s}\right|^{2}+\left|V_{u b}\right|^{2}-1$, and the relative deviation from lepton universality in $\pi^{+} \rightarrow \ell^{+} v_{\ell}(\gamma)$ decays $(\ell=e, \mu)$ by $\Delta_{e / \mu}$, one finds for the minimal supersymmetric standard model (MSSM) the results in Fig. 5.

\section{CONCLUSIONS}

Precision tests have reached per-mille and sub per-mille accuracy in derived quantities. The data are in very good agreement with the SM with the only tantalizing deviation sitting in $a_{\mu}$. When combined with the absence of any observation challenging the SM at the LHC, this provides tight constraints on new physics and it becomes increasingly likely that its energy scale is separated from the SM by at least a little hierarchy. 


\section{ACKNOWLEDGMENTS}

It is a pleasure to thank the organizers of CIPANP 2012 for the invitation to an enjoyable conference. I would also like to thank Sky Bauman, Leo Bellantoni, Jonathan Heckman, Paul Langacker, and Michael Ramsey-Musolf for collaboration on some of the topics presented here. This work was supported by the CONACyT projects 82291-F and 15 1234.

\section{REFERENCES}

1. S. L. Glashow, Nucl. Phys. 22, 579-588 (1961).

2. S. Weinberg, Phys. Rev. Lett. 19, 1264-1266 (1967).

3. C. Y. Prescott et al., Phys. Lett. B77, 347-352 (1978).

4. T. Aaltonen et al. (CDF and DØ Collaborations), arXiv:1207.1069 [hep-ex].

5. Y. Zeng (for the CDF Collaboration) and D. Boline (for the DØ Collaboration), these proceedings.

6. D. Kawall (for the Muon g-2 Collaboration), these proceedings.

7. D. Hertzog (for the MuLan Collaboration), these proceedings.

8. T. van Ritbergen and R. G. Stuart, Nucl. Phys. B564, 343-390 (2000).

9. A. Sirlin, Phys. Rev. D22, 971-981 (1980).

10. J. Erler and P. Langacker, Electroweak Model and Constraints on New Physics, in Ref. [11].

11. J. Beringer et al. (Particle Data Group), Phys. Rev. D86, 010001 (2012).

12. P. A. Baikov, K. G. Chetyrkin and J. H. Kühn, Phys. Rev. Lett. 101, 012002 (2008).

13. M. Beneke and M. Jamin, JHEP 0809, 044 (2008).

14. F. Le Diberder and A. Pich, Phys. Lett. B286, 147-152 (1992).

15. M. Davier et al., Eur. Phys. J. C56, 305-322 (2008).

16. D. Boito et al., Phys. Rev. D85, 093015 (2012).

17. J. Alcaraz et al. (ALEPH, DELPHI, L3, OPAL and LEP EW Working Group), hep-ex/0612034.

18. M. Davier, A. Höcker, B. Malaescu and Z. Zhang, Eur. Phys. J. C71, 1515 (2011).

19. A. B. Arbuzov, E. A. Kuraev, N. P. Merenkov and L. Trentadue, JHEP 9812, 009 (1998).

20. R. Beminiwattha, K. Myers, F. Nuruzzaman and P. Wang (for the Qweak Collaboration), these proceedings.

21. M. Gorchtein and W. Melnitchouk, these proceedings.

22. D. McNulty (for the MOLLER Collaboration), these proceedings.

23. R. McKeown, these proceedings.

24. P. L. Anthony et al. (SLAC-E158 Collaboration), Phys. Rev. Lett. 95, 081601 (2005).

25. D. Wang (for the PVDIS Collaboration), these proceedings.

26. J. Erler and M. J. Ramsey-Musolf, Phys. Rev. D72, 073003 (2005).

27. G. Aad et al. (ATLAS Collaboration), Phys. Rev. D86, 032003 (2012).

28. S. Chatrchyan et al. (CMS Collaboration), Phys. Lett. B710, 26-48 (2012).

29. C. S. Wood et al., Science 275, 1759-1763 (1997).

30. V. A. Dzuba, J. C. Berengut, V. V. Flambaum and B. Roberts, arXiv:1207.5864 [hep-ph].

31. S. G. Porsev, K. Beloy and A. Derevianko, Phys. Rev. D82, 036008 (2010).

32. ALEPH, DELPHI, L3 and OPAL Collaborations and LEP Working Group for Higgs Boson Searches, Phys. Lett. B565, 61-75 (2003).

33. CDF, D $\varnothing$ and Tevatron New Phenomena and Higgs Working Group, arXiv:1203.3774 [hep-ex].

34. J. Erler, arXiv:1201.0695 [hep-ph].

35. E. Kuflik, Y. Nir and T. Volansky, arXiv:1204.1975 [hep-ph].

36. A. Dighe, D. Ghosh, R. M. Godbole and A. Prasath, Phys. Rev. D85, 114035 (2012).

37. M. Buchkremer, J. M. Gérard and F. Maltoni, JHEP 1206, 135 (2012).

38. L. Bellantoni, J. Erler, J. J. Heckman and E. Ramirez-Homs, arXiv:1205.5580 [hep-ph].

39. M. E. Peskin and T. Takeuchi, Phys. Rev. D46, 381-409 (1992).

40. K. Jungmann, G. S. Giri and O. O. Versolato, AIP Conf. Proc. 1441, 552-554 (2012).

41. S. Bauman, J. Erler and M. J. Ramsey-Musolf, arXiv:1204.0035 [hep-ph]. 